\title{
Tracked Ultrasound Elastography (TrUE)
}

\author{
Pezhman Foroughi ${ }^{1}$, Hassan Rivaz ${ }^{1}$, Ioana N. Fleming ${ }^{1}$, \\ Gregory D. Hager ${ }^{1}$, and Emad M. Boctor ${ }^{1,2}$
}

1 Dept. of Computer Science, Johns Hopkins University, Baltimore, MD, USA

2 Dept. of Radiation Oncology, Johns Hopkins University, Baltimore, MD, USA

\begin{abstract}
This paper presents a robust framework for freehand ultrasound elastography to cope with uncertainties of freehand palpation using the information from an external tracker. In order to improve the quality of the elasticity images, the proposed method selects a few image pairs such that in each pair the lateral and out-of-plane motions are minimized. It controls the strain rate by choosing the axial motion to be close to a given optimum value. The tracking data also enables fusing multiple strain images that are taken roughly from the same location. This method can be adopted for various trackers and strain estimation algorithms. In this work, we show the results for two tracking systems of electromagnetic (EM) and optical tracker. Using phantom and ex-vivo animal experiments, we show that the proposed techniques significantly improve the elasticity images and reduce the dependency to the hand motion of user.
\end{abstract}

Keywords: Ultrasound, Elastography, Elasticity, Tracking, Strain.

\section{Introduction}

Ultrasound elastography is an emerging medical imaging modality which involves imaging the mechanical properties of tissue and has numerous clinical applications. Among many variations of ultrasound elastography [1, our work focuses on real-time static elastography, a well-known technique that applies quasi-static compression of tissue and simultaneously images it with ultrasound. Within many techniques proposed for static elastography, we focus on freehand palpation elasticity imaging which involves deforming the tissue by simply pressing the ultrasound probe against it. Freehand ultrasound elastography has shown great potential in clinical applications especially for diagnosis and screening of breast lesions [2. The application of elastography is not limited to breast, and other applications such as diagnosis of prostate cancer, monitoring ablation and deep vein thrombosis have also been studied.

Despite the reports on success of elastography, yet it has not become a part of any routine clinical application. The main reason is that elastography is highly qualitative and user-dependent. The best result is achieved when the user compresses and decompresses the tissue uniformly in the axial direction with the proper hand motion. It is difficult to control the compression rate as it is governed by the hand motion and the frame rate of RF data. Also, small lateral 
or out-of-plane motions can compromise the quality of images. However, it is difficult to induce pure axial motion with freehand compression. Sophisticated algorithms can only partially address the problem by compensating for in-plane motions and applying smoothness constraints. The images are also hard to interpret, and artifacts - caused by failure of the strain estimation algorithm or poor hand motion- may be mistaken for lesions inside the soft tissue. Developing an elastography technique that is not affected by poor hand motion and other sources of signal decorrelation will pave the way for wide-spread clinical use of elastography.

To improve the reliability, quality metrics such as persistence in strain images have been developed 34. This quality indicator is calculated for each image and provided to the user as feedback. Persistence is also used to merge multiple elasticity images together 3. To measure the persistence, strain is computed for two pairs of echo frames, and the resulting images are correlated. Although these techniques offers a major advantage, there remains several limitations. First, the strain has to be estimated before the calculation of the quality metric. With typical ultrasound settings, the frame rate can reach more than $30 \mathrm{~Hz}$. For subsequent frames, an efficient implementation of this image-based metric might cope with this rate. Nonetheless, the task will be extremely difficult to try all the combinations in a series of frames. Moreover, the quality metric will not be able to provide feedback to the user whether he/she should adjust the palpation in certain direction. Also, there would be minimal control over the strain rate.

The ultrasound probe is often tracked in navigation/guidance systems to provide spatial information, to form freehand $3 \mathrm{D}$ ultrasound, or to facilitate multimodality registration. In this work, we exploit the tracking data to enhance the quality of the elasticity images. We use the tracking data to select multiple image pairs that contain the optimum deformation for the elastography algorithm. The optimum value for lateral and out-of-plane motions is zero, and the optimum axial motion is determined by the specific elastography algorithm used, which is Normalized Cross-Correlation (NCC) in this work. Next, we fuse the strain images obtained from the multiple image pairs together based on the location of each strain image to improve image quality. We assume that the ultrasound data is 2D. Nonetheless similar techniques proposed here could be extended to $3 \mathrm{D}$ ultrasound.

\section{Methodology}

Consider a sequence of RF data collected during the palpation of tissue using a tracked transducer. We have previously shown that it is possible to synchronize the $\mathrm{RF}$ frames with the tracking information relying only on the same data collected during palpation [5]. From synchronization, the tracking information is interpolated at the incident time of each frame. The input to our algorithm is then a series of RF frames along with their corresponding transformation.

First, we need to define a distance function between two frames of RF data. For this purpose, we use a model of image decorrelation in presence of out-ofplane and lateral motion. RF signal is often modeled as the collective response 
of scatterers randomly distributed within the resolution cell of the ultrasound 667. Each scatterer is assumed to have an amplitude governed by the shape of the resolution cell and a phase which is distributed from 0 to $\pi$ uniformly at random. Considering a Gaussian shape for the resolution cell Prager et. al [8] calculated the correlation as a function of out-of-plane motion to be $\exp \left(-\frac{\delta^{2}}{2 \sigma^{2}}\right)$. $\delta$ and $\sigma$ denote the displacement and the width of the resolution cell respectively. Although this function is only valid for fully developed speckle, it provides a convenient estimate of correlation. It should be noted that in [8], the displacement is estimated from correlation, whereas here, we intend to define an energy function based on displacement. Extending this formula to both out-of-plane and lateral displacements, we define our energy function, $E(x, z)$, as follows:

$$
E\left(D_{x}, D_{z}\right)=\exp \left(-K_{x} \cdot D_{x}^{2}-K_{z} \cdot D_{z}^{2}\right),
$$

where $D_{x}$ and $D_{z}$ represent the displacement in out-of-plane and lateral directions. $E$ does not depend on axial motion $\left(D_{y}\right)$ since displacement in axial direction is necessary for strain estimation. $K_{x}$ and $K_{z}$ determine the sensitivity to a certain direction. In order to be able to use this function, we need a component-wise metric representing the distance of two frames given their homogeneous transformations. The first step is to compute the relative transformation between them. Suppose $a=\left[\begin{array}{lll}a_{x} & a_{y} & a_{z}\end{array}\right]^{T}$ is the axis-angle representation of the relative rotation, and $t=\left[t_{x} t_{y} t_{z}\right]^{T}$ is the relative translation. Assuming a small rotation, the relative displacement of a point, $P=[x y 0]^{T}$, will be $d=a \times P+t$. We then define the distance vector of two frames, $D=\left[D_{x} D_{y} D_{z}\right]^{T}$, as the RMS of the components of $d$ for all the points in the region of interest (ROI):

$$
\begin{aligned}
& D_{x}=\operatorname{sqrt}\left\{\frac{1}{\left(y_{2}-y_{1}\right)} \int_{y_{1}}^{y_{2}}\left(-a_{z} \cdot y+t_{x}\right)^{2} d y\right\} \\
& D_{y}=\operatorname{sqrt}\left\{\frac{1}{\left(x_{2}-x_{1}\right)} \int_{x_{1}}^{x_{2}}\left(a_{z} \cdot x+t_{y}\right)^{2} d x\right\} \\
& D_{z}=\operatorname{sqrt}\left\{\frac{1}{\left(y_{2}-y_{1}\right)\left(x_{2}-x_{1}\right)} \int_{x_{1}}^{x_{2}} \int_{y_{1}}^{y_{2}}\left(a_{x} \cdot y-a_{y} \cdot x+t_{z}\right)^{2} d y d x\right\}
\end{aligned}
$$

where $\operatorname{sqrt}\{$.$\} returns the root. Here, ROI is assumed to be rectangular and$ determined by $x_{1}, x_{2}, y_{1}$, and $y_{2}$. The vector $D$ provides a measure of distance for each direction separately. We use this vector in Equation (1) which gives us an estimate of "pseudo-correlation" over the ROI.

The data goes through four stages of processing to create a single high-quality strain image. In the first step, few images are selected from the data series that are approximately collected from one cross-section of tissue with minimal lateral and out-of-plane motion. To this end, the energy function of each frame is computed with respect to all other frames in the sequence. Then, the total energy is found for each frame as the sum of the energies of the $M$ closest frames, where closeness implies higher energy, and $M$ is the maximum number of frames to be selected. Then, the frame with the highest total energy (the center frame) 
is identified, and the $M$ closest frames to the center frame including itself are selected. Additionally, the frames that have $E$ of less than 0.5 with respect to the center frame are disqualified. This is applied to ensure lower number of frames are chosen when $M$ frames from one cross-section are not available.

In the next stage, the program evaluates all possible combination of frame pairs for elastography. For $M$ frames, there will be $\left(\begin{array}{c}M \\ 2\end{array}\right)=M(M-1) / 2$ pair combinations which will be compared using a slightly modified version of $E$. Since the pairs are directly compared, it suffices to minimize the exponent of Equation (1) in order to maximize $E$. We also add a term for axial motion that penalizes compressions that are higher than an optimum compression value, $t_{\text {opt }}$. Hence, a "cost function", $C 1$, is defined as follows:

$$
C 1(D)=K_{x} \cdot D_{x}^{2}+K_{y} \cdot{\tilde{D_{y}}}^{2}+K_{z} \cdot D_{z}^{2}, \quad \tilde{D}_{y}= \begin{cases}D_{y}-t_{\text {opt }}, & \left|D_{y}-t_{\text {opt }}\right|>0 \\ 0, & \left|D_{y}-t_{\text {opt }}\right| \leq 0\end{cases}
$$

where $t_{\text {opt }}$ implies the optimal strain, which can be theoretically defined as described in [9]. Here, $t_{\text {opt }}$ is set depending on the robustness of the elasticity estimation algorithm. Its value might be within the range of the resolution of the tracker. Therefore, at this stage we do not assign a penalty for the compressions less than $t_{o p t}$. If the compression is close to zero, the contrast of the reconstructed image degrades. The program filters the pairs with low compression in the next stage using image content. Similar to the first part, a maximum number of frames with lowest cost are selected provided that the cost is lower than a threshold. The threshold is not strict to ensure acceptable pairs are not filtered.

The final pairs are selected by recovering the global lateral motion and compression by matching the two RF frames in each pair. The tracking information is used to initialize the search. For instance, the search range for compression is set to be from zero to the tracker reading in axial direction padded in both sides with the maximum error of the tracker. Given two frame $I_{1}$ and $I_{2}$, the amount of lateral motion $a$, and compression, $b$, is found by solving cross-correlation:

$$
\arg \max _{a, b}\left\{\sum_{x, y \in G} I_{1}(x, y) \cdot I_{2}(x+a, b y)+I_{1}(x-a,-b y) \cdot I_{2}(x, y)\right\} .
$$

The RF data is normalized with standard variation and assumed to have zero mean. We employ two tricks which extensively increases the speed of search. First, we do not match the entire image to solve for these parameters. Instead, only pixels on a grid, $G$, are used as described by Equation (44). The two terms of Equation (4) ensures that the search remains reciprocal, which means switching the images only affects the sign of $a$ and $b$. Second, $a$ is recovered by matching only the top part of the two images while $b$ is fixed to one. The reason is that the displacement due to compression is minimal in that region.

Having the global motions, the cost function is modified to penalize very low compressions: 


$$
C 2(\widehat{D})=K_{x} \cdot{\widehat{D_{x}}}^{2}+K_{y} \cdot \frac{\left|\widehat{D_{y}}-t_{\text {opt }}\right|^{3}}{\widehat{D_{y}}+c}+K_{z} \cdot D_{z}^{2},
$$

where $\widehat{D_{x}}$ and $\widehat{D_{y}}$ are the global motions from Equation (4) converted to mm. $c$ is a small number that limits the cost of zero compression. Finally, the pairs with the lowest cost are selected until a maximum number of frame pairs is reached or the minimum cost grows higher than the average cost.

The last step involves computing the strain for all the selected frame pairs. We have implemented normalized cross-correlation (NCC) [10 to recover the displacements and least squares estimation to calculate the strain. Before calculating strain, the global lateral motion and compression from the previous step are compensated in one image using cubic interpolation. This is known to reduce the error of strain estimation [11. The final strain image, $S_{\text {final }}$ is the weighted average of all the strains:

$$
S_{\text {final }}=\frac{\sum_{i=1}^{m} w_{i} \cdot S_{i}}{\sum_{i=1}^{m} w_{i}}, w_{i}= \begin{cases}\frac{\rho_{i}}{1-\rho_{i}}, & \rho_{i}>0.7 \\ 0, & \text { otherwise }\end{cases}
$$

where $\rho_{i}$ is the correlation coefficient for the $i$ th pair after applying the displacements, and $m$ is the number of pairs. Fusing the strains in this fashion is acceptable since the algorithm only allows for compressions that are close to a predetermined amount optimal for strain estimation.

\section{Experiments and Results}

We acquired ultrasound data using a SONOLINE Antares ${ }^{\mathrm{TM}}$ ultrasound system (Siemens Medical Solutions USA, Inc.) with a high-frequency ultrasound transducer (VF10-5) at center frequency of 6-8 MHz. We accessed RF through the Axius Direct ${ }^{\mathrm{TM}}$ Ultrasound Research Interface provided by Siemens. Our custom data acquisition program was connected to this interface to send the command for capturing RF data. At the same time, the program collected tracking information from either a "Polaris" optical tracker (Northern Digital Inc., Waterloo, Canada) with passive markers or the "medSAFE" EM tracker (Ascension Tech. Corp.).

$\mathrm{RF}$ data and tracking information was captured from a breast phantom containing a harder lesion (CIRS elastography phantom, Norfolk, VA) and ex-vivo pig liver. Alginate was injected to the liver to mark a part of liver, and then, that area was ablated. The users were asked to palpate the tissue over the hard lesion in the breast phantom and the ablated lesion in the pig liver while data was being collected. Between 100 to $138 \mathrm{RF}$ frames were acquired with the rate of about 30 frames per second.

The first set of data was captured by an experienced user from the breast phantom. Figure 1(a) shows the translation components of hand motion with respect to the first frame. The axial motion is dominant and there is only a gradual drift in the lateral and elevational directions. Figure 1(b) depicts the high-quality strain image resulting from the TrUE algorithm. 


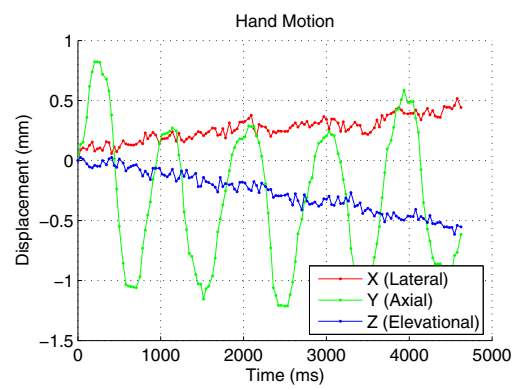

(a) Freehand palpation

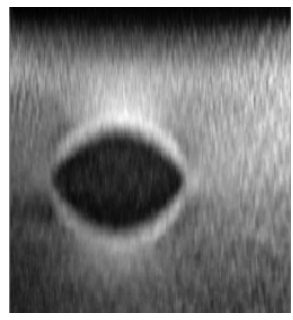

(b) Strain image (TrUE)

Fig. 1. (a) shows the translation of probe w.r.t. the first image. Proper hand motion is applied as the axial compression is dominant. (b) is the output of our proposed algorithm.

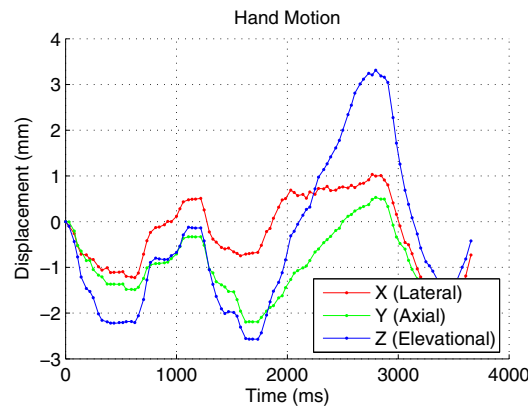

(a) Poor palpation with EM tacking

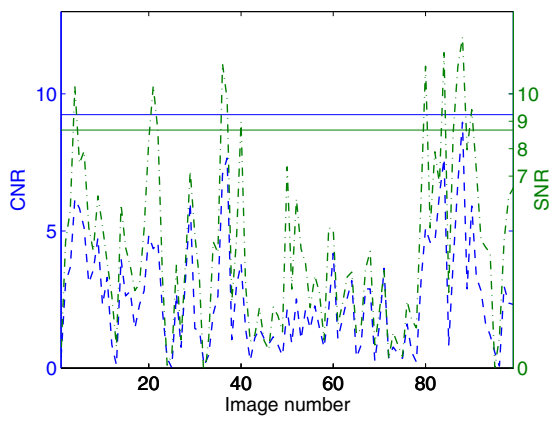

(c) Consecutive vs. TrUE

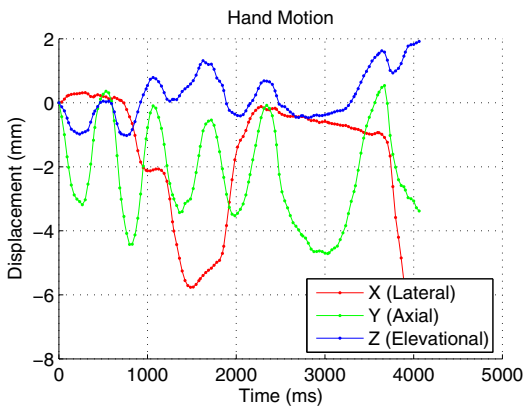

(b) Poor palpation with optical tracking

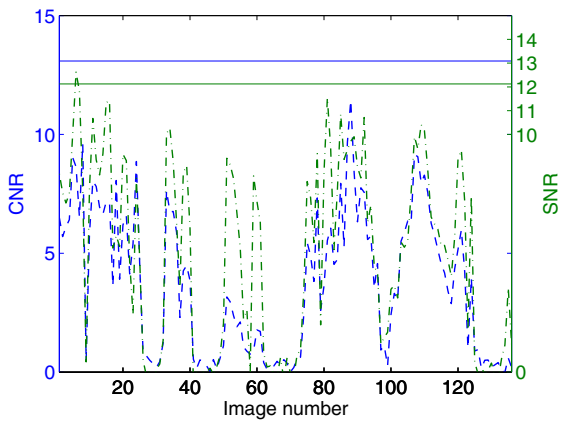

(d) Consecutive vs. TrUE

Fig. 2. Two cases of improper motions are shown where the hand motion suffers from large lateral and elevational components evident in relative translations. The results of case 1 with EM tacker is shown on the left column, and the results of case 2 with optical tracker is shown on the right column. 
Applying a compression similar to the one shown in Figure 1(a) is a difficult task for novice or even intermediate users. This is especially the case where axial compression does not translate into a simple up and down motion. Ultrasound gel creates a slippery surface that makes the palpation prone to out-of-plane motion. Two case are shown in Figure 2, where one is tracked with the EM tracker and the other one with the optical tracker. In Figure 2(a) the hand motion contains a large amount of out-of-plane motion, whereas, in Figure 2 (b), the user has moved the probe laterally. In both cases, the TrUE algorithm generates reliable results. Figures 2 (c) and (d) show the contrast-to-noise ratio (CNR) and signal-to-noise ratio $(\mathrm{SNR})$ of the strain image. The $\mathrm{CNR}$ and $\mathrm{SNR}$ value are computed from:

$$
\mathrm{CNR}=\sqrt{\frac{2\left(\bar{s}_{b}-\bar{s}_{t}\right)^{2}}{\sigma_{b}^{2}+\sigma_{t}^{2}}}, \quad \mathrm{SNR}=\frac{\bar{s}}{\sigma},
$$

where $\bar{s}$ and $\sigma$ denote the mean and standard deviation of intensities. The $t$ or $b$ subscripts show that the computation is only for the target or the background region, respectively. The SNR and CNR for computing the strain from consecutive frames (the dashed curve) is compared to the SNR and CNR of the strain image from the proposed method (solid line). Using consecutive frames is the standard method of elastography in ultrasound machines. Almost in all cases the TrUE algorithm outperforms the consecutive frames by a large margin.

Although the SNR and CNR provide quantitative measures to compare the strain images, they do not directly reflect the visual quality of strain. In Figure 3 , we show results of elastography using our frame selection technique as well as four other strain images calculated from consecutive frames. The Figure shows the effects of improper compression in consecutive frames in the strain image. At the same time our algorithm provides a single reliable strain.

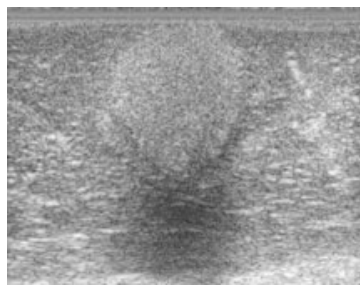

(a) B-mode

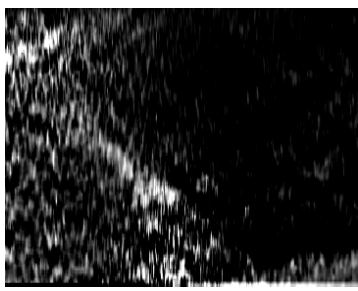

(d) $2 \& 3$

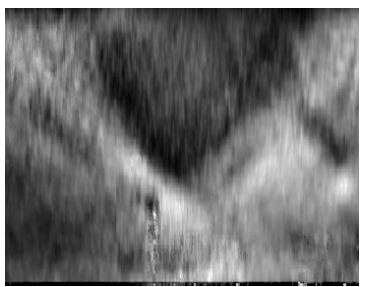

(b) $\operatorname{TrUE}$

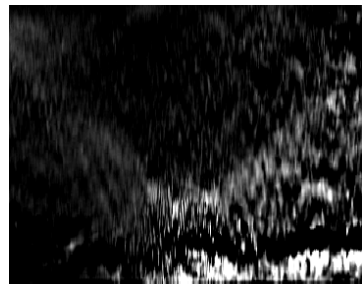

(e) $3 \& 4$

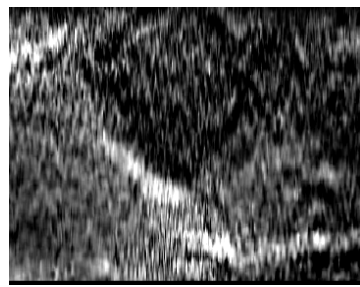

(c) $1 \& 2$

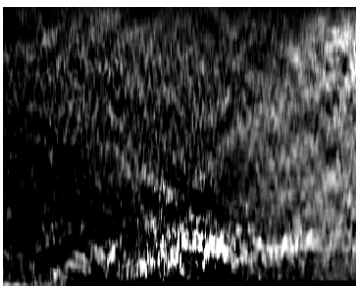

(f) $4 \& 5$

Fig. 3. Comparison of the strain from TrUE vs. consecutive frames for ex-vivo pig liver 


\section{Discussion}

We presented a method of ultrasound elastography which is robust to the quality of the hand motion of the user. Using the information from an external tracker, it automatically selects multiple frame pairs with a specific compression and minimal undesired motions. Our approach does not take into account the tissue motion from other sources such as breathing or patient motion. However, these types of motions are not normally problematic since they occur with a slower pace compared to hand motion.

Our experiments shows that even when the transducer has severe lateral or out-of-plane motions, the algorithm still manages to produce good results. The multi-stage frame selection and careful image fusion makes the TrUE method less sensitive to tacker accuracy and robust to strain estimation failures.

We are planning to use the proposed method in a breast cancer study. For this purpose, we will be implementing our MATLAB code in C. The strain estimation which is still the bottleneck of our approach will be executed in GPU allowing for the use of sophisticated algorithms.

Acknowledgments. Pezhman Foroughi, Hassan Rivaz, and Ioana Fleming are supported by the U.S. Department of Defense pre-doctoral fellowship program.

\section{References}

1. Ophir, J., Alam, S., Garra, B., Kallel, F., Konofagou, E., Krouskop, T., Varghese, T.: Elastography: ultrasonic estimation and imaging of the elastic properties of tissues. Annu. Rev. Biomed. Eng. 213, 203-233 (1999)

2. Garra, B., et al.: Elastography of breast lesions: initial clinical results. Radiology 202, 79-86 (1997)

3. Lindop, J.E., Treece, G.M., Gee, A.H., Prager, R.W.: An intelligent interface for freehand strain imaging. Ultrasound Med. Biol. 34, 1117-1128 (2008)

4. Jiang, J., Hall, T.J., Sommer, A.M.: A novel strain formation algorithm for ultrasonic strain imaging. In: IEEE Ultrasonics Symposium, pp. 1282-1285 (2006)

5. Foroughi, P., Hager, G., Boctor, E.: Robust elasticity imaging using external tracker. In: IEEE Int. Symp. Biomed. Imag., pp. 209-212 (2009)

6. Wagner, R., Smith, S., Sandrik, J., Lopez, H.: Statistics of Speckle in Ultrasound B-Scans. IEEE Trans. Sonics and Ultrasonics 17(3), 251-268 (1983)

7. Shankar, P.: A general statistical model for ultrasonic backscattering from tissues. IEEE Trans. Ultrason. Ferroelectr. Freq. Control 47(3), 727-736 (2000)

8. Prager, R., et al.: Sensorless freehand 3-d ultrasound using regression of the echo intensity. Ultrasound Med. Biol. 29, 437-446 (2003)

9. Varghese, T., Ophir, J.: A theoretical framework for performance characterization of elastography: the strain filter. IEEE Transactions on Ultrasonics, Ferroelectrics and Frequency Control 44, 164-172 (1997)

10. Céspedes, I., Huang, Y., Ophir, J., Spratt, S.: Methods for estimation of subsample time delays of digitized echo signals. Ultrasound Imaging 17(2), 142-171 (1995)

11. Varghese, T., Ophir, J.: Performance optimization in elastography: Multicompression with temporal stretching. IEEE Transactions on Ultrasonics, Ferroelectrics and Frequency Control 18(22), 193-214 (1996) 